\title{
The effect of intra-articular ozone injection on pain and physical function in knee osteoarthritis: A prospective study
}

\author{
Gülçin Gazioğlu Türkyılmaz ${ }^{1}$, Mesut Bakır ${ }^{2}$, Şebnem Rumeli Atıcı ${ }^{1}$ \\ ${ }^{1}$ Division of Algology, Mersin University School of Medicine, Mersin, Turkey \\ ${ }^{2}$ Division of Algology, Mersin City Education and Research Hospital, Mersin, Turkey
}

Received: 2020-08-09.

Accepted: 2020-08-26

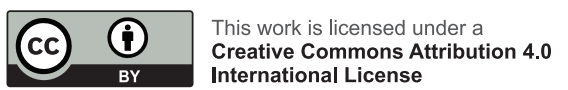

J Clin Med Kaz 2020; 6(60):68-72

Corresponding author:

Gülçin Gazioğlu Türkyılmaz.

E-mail: drgulcint@gmail.com;

ORCID: 0000-0003-4148-1958

\section{Abstract}

Introduction: This study aims to evaluate the effect of intraarticular ozone treatment on pain and physical functionality by measuring Numerical Pain Rating Scale (NRS), WOMAC Osteoarthritis Index (WOMAC-OI), and Active Joint Range of Motion (AROM) in patients diagnosed with osteoarthritis.

Material and methods: A total of 30 patients diagnosed with stage 2-3 osteoarthritis according to the Kellgren-Lawrence Classification System (K-L) were included in the study. Ozone gas was administered (10 $\mathrm{ml}$ of $10 \mathrm{mcg} / \mathrm{ml}$ per session) twice weekly for a total of four sessions. AROM examination was performed at pre-treatment baseline (BL), post-treatment (PT), and two weeks after treatment (PT2). During these periods, NRS was assessed and WOMAC-OI questionnaire was administered.

Results: Significant improvement in NRS and WOMAC-OI total index scores were observed at PT and PT2 periods compared to BL

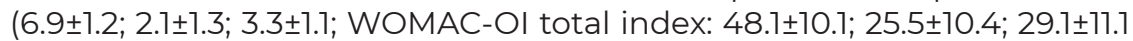
respectively; $\mathrm{p}<0.001$ for all). However, in both of these evaluation methods, the recovery at PT2 had decreased compared to the PT period (NRS $p<0.001$; WOMAC-OI $p<0.05$ ). Significant improvement was observed in the AROM values measured during the PT and PT2 periods, compared to baseline (105.6 $\pm 10.4^{\circ} ; 118.8 \pm 8.1^{\circ} ; 117.5 \pm 9.2^{\circ}$, respectively) $(p<0.001)$. There was no difference between the PT and PT2 periods in terms of AROM measurements ( $p>0.05$ ).

Conclusion: Our study demonstrates that intra-articular ozone injection treatment provides improvement in pain and physical functionality in osteoarthritis patients. In our two-month follow-up assessment, although evaluation methods indicated that there was regression in recovery, AROM measurements showed that there was no regression in function. Therefore, we believe that future studies may include AROM as an objective measurement among evaluation methods.

Key words: range of motion, ozone, intra-articular injection, numerical rating scale, osteoarthritis, WOMAC osteoarthritis index

\section{Introduction}

Osteoarthritis (OA) is the most common degenerative joint disease, characterized by progressive cartilage damage and deterioration of the joint structure due to inflammation in the synovium and cartilage structure. Treatment of the damaged joint cartilage is very challenging due to its limited self-recovery capacity. Today, there is still no definite treatment method that can delay the structural progression of OA or postpone the need for total knee replacement [1]. However, some conservative treatments, including modification of lifestyle activities, analgesics, and physical therapy to reduce symptoms may be applied. Patients who do not benefit from these methods are offered intra-articular hyaluronic acid (HA), platelet-rich plasma (PRP), and steroids, as well as ozone injections among these treatment modalities [2-4]. 
In various stress conditions, several inflammatory mediators, including reactive oxygen species such as nitric oxide and hydrogen peroxide, are produced by chondrocytes and cause cartilage damage [5]. Ozone therapy is believed to act by creating controlled oxidative stress, activating the body's own antioxidant systems, and reducing the concentration of proinflammatory cytokines such as TNF $\alpha$ which are largely responsible for joint and bone damage $[6,7]$. Intra-articular ozone injections have been used to improve clinical symptoms such as pain and limited range of motion in the initial stages of OA [8-10].

In patients undergoing intra-articular ozone injections for knee osteoarthritis, Visual Analogue Scale (VAS) and WOMACOI are commonly used to evaluate the treatment's efficacy. However, the inclusion of test data such as Active Joint Range of Motion (AROM), in which the change of function of the knee joint is evaluated by the doctor, may better demonstrate the effectiveness of ozone therapy. We did not find any study in the literature evaluating the intra-articular ozone injection treatment of the knee joint using ROM, together with questionnaires WOMAC-OI $[11,12]$. This study aims to evaluate the effect of intra-articular ozone injections on pain and function by using Numerical Pain Rating Scale (NRS), WOMAC-OI, and Active Joint Range of Motion (AROM) in patients diagnosed with stage 2-3 OA according to the Kellgren-Lawrence Classification System (K-L).

\section{Material and methods}

This study received approval from the local ethics committee (Date: 18/09/2019; No. 2019/409). Thirty-six knee osteoarthritis patients who applied to the algology outpatient clinic with knee pain were included in the study. Six patients were excluded from the study (Figure 1). A total of 30 patients

Figure 1 - Flow chart of the study.

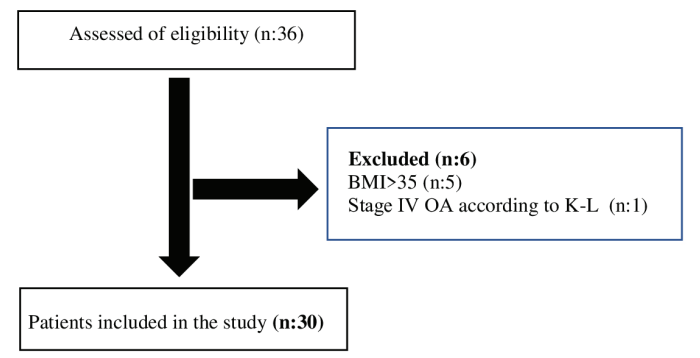

between the ages of 18-80 who were diagnosed with stage 2-3 osteoarthritis according to the Kellgren-Lawrence Classification System (K-L) [13], who applied to the Algology outpatient clinic due to knee pain between the dates of 01/10/2019 - 01/02/2020 and who consented to intra-articular ozone injection therapy were included in the study. Patients who were diagnosed with Stage 1 and 4 OA according to K-L Classification, with history of knee trauma within the past month, morbid obese patients with body mass index (BMI) of $>35$, local or systemic infection, bleeding diathesis, and those who did not consent to completing the WOMAC-OI questionnaire were excluded from the study. None of the patients included in the study were receiving nonsteroidal anti-inflammatory drugs or corticosteroids, and they did not receive it during the study. In addition, none of the patients received any other intra-articular knee injections within the month prior to starting ozone injection treatment.
Patients underwent two sessions a week for a total of four sessions of intra-articular knee ozone therapy. Contrast medium was injected into the joint space and the appropriate injection site was confirmed with fluoroscopy before ozone injection. Patients received $10 \mathrm{ml}$ of $10 \mathrm{mcg} / \mathrm{ml}$ ozone as intraarticular injections (administered dose and concentration was determined to be in accordance with the Madrid Declaration [14]). Age, gender, and BMIs of the patients were recorded before treatment. NRS scores of the patients were evaluated at pretreatment baseline (BL), at the end of treatment (PT), and at two-month follow-up (PT2). In order to evaluate functional status, the WOMAC-OI questionnaire, which has achieved Turkish validity and reliability, was completed by patients at all three periods [15]. Furthermore, Active Joint Range of Motion (AROM) of the patients was measured with goniometry at the same three periods.

\section{WOMAC osteoarthritis index}

The index consists of a total of 24 items, in which five items evaluate pain, two evaluate stiffness, and 17 evaluate physical function. Total score as well as separate scores of subgroups can be evaluated. Maximum score is 20 for the pain subgroup, 8 for stiffness, and 68 for physical function. High scores are indicative of poor values.

\section{Active range of motion}

Patients in supine position were asked to bring their hip joints to neutral position and when in $<90^{\circ}$, to bring their knees to flexion. Measurements were made by placing the goniometer's axis on the lateral epicondyle of the femur, with one arm oriented on the greater trochanter and the other on the lateral malleolus (Figure 2). Goniometric measurement of all patients was conducted by the same person.

Figure $\mathbf{2}$ - Active range of motion measurement of the knee

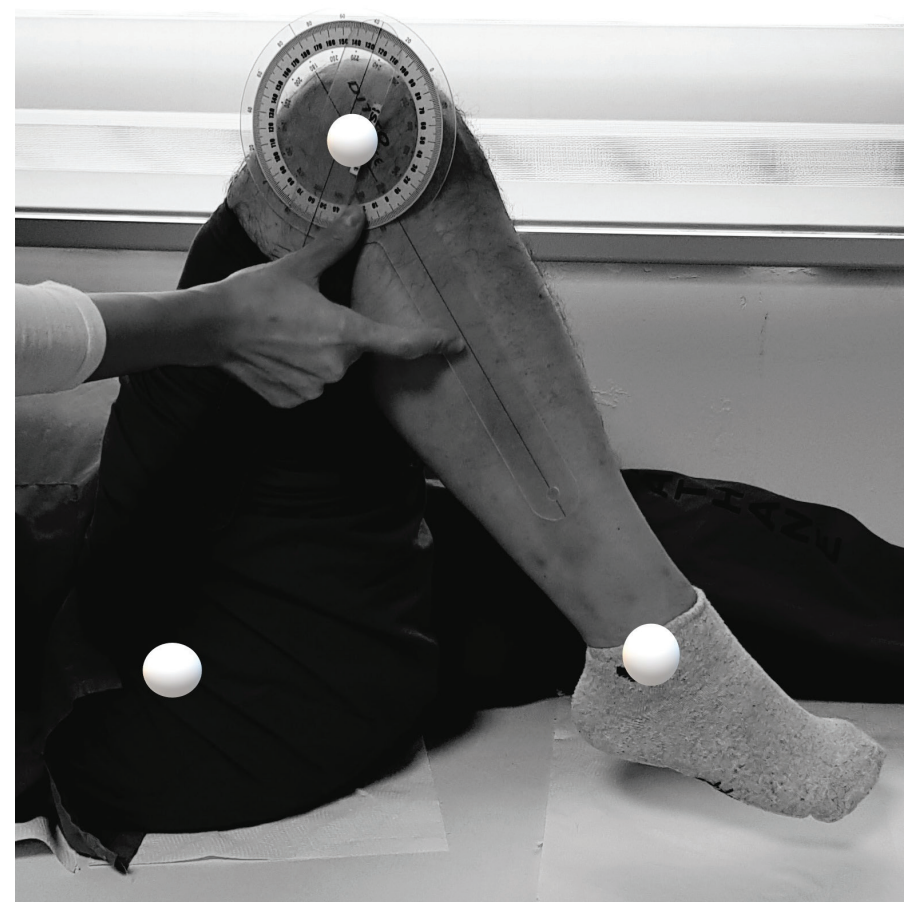

\section{Statistical analysis}

Statistical analysis of the data was conducted using the SPSSv.24 "Statistical Package for the Social Sciences version 24". Descriptive statistics were used for categorical variables and frequency calculations were expressed as percentage. Paired 
Samples t-test was used to compare the K-L Classification results of the patients. A p value of $<0.05$ was considered statistically significant.

\section{Results}

Mean age of the patients was $62.0 \pm 9.4$ (min: 44; max: 79) and mean BMI was $29.0 \pm 2.63$ (min: 22.3; max: 35). Twentyfour of the patients were females $(80 \%)$. According to K-L Classification, 17 patients were Stage 3, while 13 were Stage 2 $(43.3 \%)$.

Mean NRS was $6.9 \pm 1.2$ in the BL period. NRS scores were significantly lower in the PT $(2.1 \pm 1.3)$ and PT2 $(3.3 \pm 1.1)$ periods compared to the BL period $(\mathrm{p}<0.001)$. However, NRS was increased in the PT2 period compared to PT $(\mathrm{p}<0.001)$ (Figure 3). There was no statistically significant difference between Stage 2 and Stage 3 OA patients regarding mean NRS scores $(\mathrm{p}>0.05)$ (Table 1$)$.

The change in the mean values of WOMAC-OI total scores, and the subparameters of pain, stiffness and function throughout the evaluation process are provided in Figure 4.

Figure 3 - NRS scores of the study patients

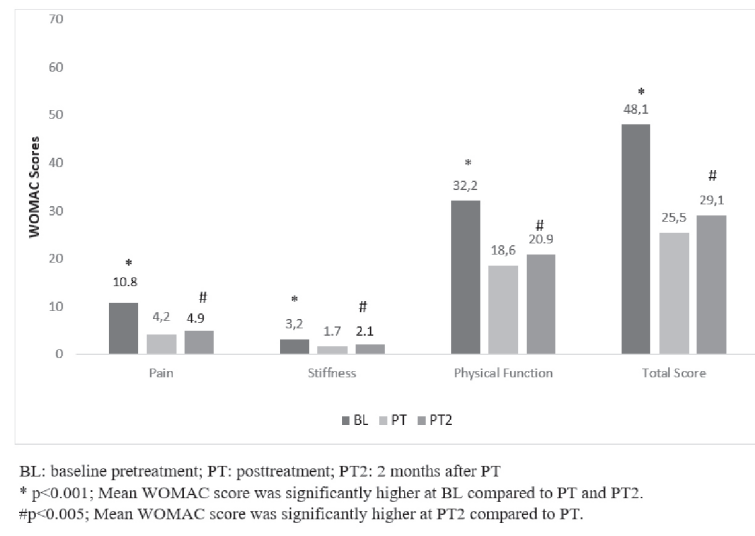

Figure 4 - Change in mean WOMAC-OI total scores and sub parameters at BL, PT, and PT2 periods.

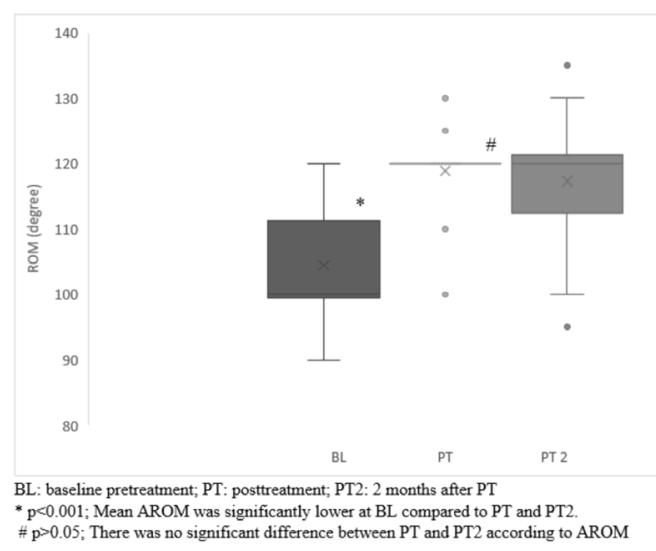

In regards to WOMAC-OI, total scores and all sub parameter scores were significantly higher at BL compared to PT and PT2 $(\mathrm{p}<0.001)$. Furthermore, mean WOMAC-OI total and mean sub parameter scores at PT2 were significantly higher in comparison to PT $(\mathrm{p}<0.05)$. According to WOMAC-OI Function scores, Stage 2 OA patients had significantly lower mean scores than Stage 3 OA patients at both PT and PT2 periods $(\mathrm{p}=0.02$, $\mathrm{p}=0.01$ ) (Table 1). According to WOMAC-OI total scores, Stage 2 OA patients had significantly lower mean scores at PT2 compared to Stage 3 OA patients $(\mathrm{p}=0.02)$ (Table 1$)$. K-L Stage 2 and Stage 3 OA patients

\begin{tabular}{|c|c|c|c|c|}
\hline & Evaluation time & $\begin{array}{l}\text { Stage } 2 \\
(\mathrm{n}: 13)\end{array}$ & $\begin{array}{l}\text { Stage } 3 \\
\text { (n:17) }\end{array}$ & $\mathrm{p}$ \\
\hline \multirow[t]{3}{*}{ NRS } & $\mathrm{BL}$ & $6.7 \pm 1.3$ & $7.1 \pm 1.2$ & 0.46 \\
\hline & PT & $2.1 \pm 1.1$ & $2.1 \pm 1.5$ & 0.94 \\
\hline & PT2 & $2.9 \pm 0.9$ & $3.6 \pm 1.1$ & 0.80 \\
\hline \multirow{3}{*}{$\begin{array}{l}\text { WOMAC-OI } \\
\text { Pain }\end{array}$} & $\mathrm{BL}$ & $11.1 \pm 4.2$ & $10.5 \pm 2.3$ & 0.64 \\
\hline & $\mathrm{PT}$ & $4.3 \pm 2.8$ & $4.1 \pm 2.3$ & 0.84 \\
\hline & PT2 & $4.4 \pm 2.5$ & $5.2 \pm 2.4$ & 0.40 \\
\hline \multirow[t]{3}{*}{ Stiffness } & $\mathrm{BL}$ & $2.6 \pm 1.3$ & $3.7 \pm 1.7$ & 0.10 \\
\hline & $\mathrm{PT}$ & $1.4 \pm 0.8$ & $1.9 \pm 1.2$ & 0.26 \\
\hline & PT2 & $1.6 \pm 0.9$ & $2.5 \pm 1.3$ & 0.06 \\
\hline \multirow[t]{3}{*}{ Function } & $\mathrm{BL}$ & $30.7 \pm 9.5$ & $33.3 \pm 5.9$ & 0.37 \\
\hline & PT & $14.9 \pm 6.5$ & $21.5 \pm 8.2$ & $0.02^{*}$ \\
\hline & PT2 & $16.8 \pm 6.2$ & $24.0 \pm 8.3$ & 0.01* \\
\hline \multirow[t]{3}{*}{ Total } & $\mathrm{BL}$ & $46.4 \pm 12.7$ & $49.4 \pm 7.8$ & 0.42 \\
\hline & PT & $21.5 \pm 8.8$ & $28.5 \pm 10.9$ & 0.07 \\
\hline & PT2 & $23.9 \pm 8.3$ & $33.2 \pm 11.4$ & $0.02^{*}$ \\
\hline \multirow[t]{3}{*}{ AROM } & $\mathrm{BL}$ & $106.5 \pm 12.1$ & $104.9 \pm 9.2$ & 0.68 \\
\hline & PT & $120.3 \pm 8.5$ & $117.6 \pm 7.9$ & 0.37 \\
\hline & PT2 & $118.4 \pm 9.2$ & $116.7 \pm 9.5$ & 0.62 \\
\hline
\end{tabular}

NRS: Numeric Rating Scale; AROM: Active Range of Motion; BL: baseline pretreatment; PT: posttreatment; PT2: 2-month follow-up

* $p<0.05$; Stage 3 OA patients had significantly higher scores than Stage 2 OA patients

Mean AROM values of the patients were determined as $105.6 \pm 10.4^{\circ}$ at $\mathrm{BL}, 118.8 \pm 8.1^{\circ}$ at $\mathrm{PT}$ and $117.5 \pm 9.2^{\circ}$ at PT2 (Figure 5). Both PT and PT2 values were significantly higher than baseline values $(\mathrm{p}<0.05)$.

Figure 5 - AROM values of the patients at BL, PT, and PT2

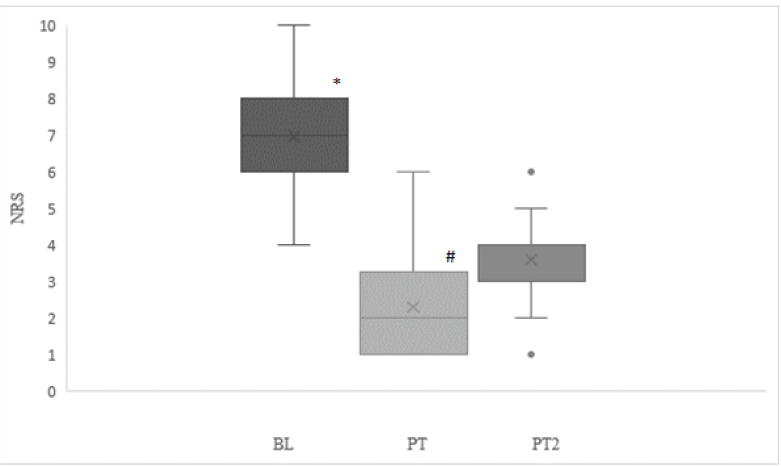

NRS: Numeric Rating Scale, BL: baseline pretreatment; PT: posttreatment; PT2: 2 months after PT * $\mathrm{p}<0.001$; Mean NRS score was significantly higher at BL compared to PT and PT2. \# $<<0.001$; Mean NRS score was significantly lower at PT compared to PT2.

When Stage 2 and Stage 3 patients were compared, no significant difference in mean AROM was observed in any of the periods $(\mathrm{p}>0.05)$ (Table 1$)$

\section{Discussion}

This study is the first to use AROM with NRS and WOMAC-OI scores to interpret the results of intra-articular ozone injection therapy in patients with C-L stage 2 and 3 OA. All three evaluation methods revealed improvement in both PT and PT2 periods compared to BL. However, while the self-assessment scales NRS and WOMAC-OI determined that recovery at PT2 had regressed compared to the PT period, AROM evaluation determined there was no significant difference in recover rate. These results showed that addition of Active Range of Motion, an objective measurement, to the evaluation process 
in the follow-up of recovery in knee function may be important. Epidemiological studies have revealed that in half of the world population aged 65 and over, the most common joint disorder is the diagnosis of OA [16]. The mean age of our patients was $62.0 \pm 9.4$ and was compatible with the literature. In one randomized controlled study evaluating the effectiveness of intra-articular knee ozone injection in patient with knee osteoarthritis, 89.6\% (n:86) of 96 knee osteoarthritis patients were reported to be women [8]. In our study, $80 \%$ of the patients were female (n: 24).

Studies have applied intra-articular ozone injections in various dosages and time intervals. In one study, $10 \mathrm{ml}$ of 30 $\mathrm{mcg} / \mathrm{ml}$ ozone was injected for 3 weeks and the effect on pain, stiffness and function was evaluated six months after treatment. Patients showed significant improvement in terms of pain, stiffness, and function according to the comparison of VAS and WOMAC-OI scores evaluated at baseline and six-month followup [3]. In another study evaluating intra-articular ozone injection treatment in 63 patients with knee OA, $10 \mathrm{ml}$ of $20 \mathrm{mcg} / \mathrm{ml}$ ozone was injected once a week for 8 weeks. Patients were evaluated with VAS scores and WOMAC-OI at baseline and 4, 8, and 16 weeks after treatment. In the aforementioned study, it was observed that the ozone group had superior VAS scores, joint function, and quality of life compared to the placebo group and improvement was continuous throughout the evaluation period [8]. In our study, patients received $10 \mathrm{ml}$ of $10 \mathrm{mcg} / \mathrm{ml}$ ozone injections twice weekly for two weeks.

Despite the lower dosage and shorter timeframe, we observed significant improvement in NRS scores and all WOMAC-OI sub parameters at the end of the 2-week treatment as well as 2 months after treatment compared to pretreatment scores. However, regression in improvement observed at twomonth follow-up leads us to believe that further studies must be conducted in regards to various dosage and reinjection periods.

One meta-analysis which examined the short-term therapeutic effects of ozone on knee osteoarthritis analyzed 8 randomized controlled studies which used both VAS and WOMAC-OI for evaluation. This analysis reported that ozone therapy had a therapeutic effect and provided short-term pain relief compared to the placebo (ozone: 355; control: 363 ). The analgesic effect is mostly observed one month after treatment and progressively fades 3-6 months after treatment [9]. Although evaluation of treatment results by the patients themselves is very important, upholding scientific study data with objective markers provide better evaluation of results. Therefore, in our study, we evaluated the effect of intra-articular ozone therapy with active EHA measurements as well as NRS and WOMAC-OI scores. We observed $\geq 50 \%$ improvement in WOMAC-OI, NRS scores and AROM measurements at post-treatment and two months after treatment in comparison to baseline values. However, although it was observed that the improvement in scores of the second month decreased compared to post-treatment, there was no decrease in improvement according to AROM measurements. We believe that this may be due to two reasons. First, although functional improvement continues, we may have observed a decrease in recovery according to results of the questionnaire, since patients do not achieve their expectations of full recovery at the end of the study period. Second, the healing effect may be due to the short-term anti-inflammatory effect of ozone, rather than its healing effect on cartilage structure. Since morphologic improvement in cartilage structure was not followed up with MRI before and after treatment, the reason could not be clearly revealed.

Although studies in the literature have compared other intra-articular injection treatments according to K-L Classification, we did not encounter a study that evaluated intra-articular ozone injection treatment. One study reported that, following PRP injections, patients with milder cartilage degeneration according to K-L Classification (stage 1-2) showed better treatment efficacy at six months compared to patients with more advanced stage cartilage degeneration (stage 3-4) [17]. In our study, patients with stage 2 OA according to K-L Classification had significantly better WOMAC-OI function and total scores compared to Stage 3 patients.

\section{Limitations}

Our study had some limitations. The long-term effect of ozone therapy could be evaluated by following the patients for a longer period (6 months i.e.). Another limitation was that changes in the morphology of cartilage structure could have been revealed in greater detail with an imaging method (MRI) before and after treatment.

In addition, before the start of treatment, it could have been explained to patients that full recovery of all symptoms was not expected, and that $>50 \%$ improvement would be considered as recovery.

\section{Conclusion}

In our study, we concluded that ozone therapy improves pain and physical functionality in patients with osteoarthritis However, further studies are needed in order to illuminate the uncertainties in dosage, number of sessions, and intervals should be implemented in intra-articular ozone injections. In addition, we believe the inclusion of active range of motion as a method of evaluation in further studies on ozone therapy, especially regarding joint diseases, is very important.

Disclosures: There is no conflict of interest for all authors.

\section{Acknowledgements: None.}

Funding: None.

\section{References}

1. Barbour KE, Helmick CG, Boring M, Brady TJ. Vital Signs: Prevalence of Doctor-Diagnosed Arthritis and Arthritis-Attributable Activity Limitation - United States, 2013-2015. MMWR Morbidity and mortality weekly report. 2017; 66(9):246-253.https://doi. org/10.15585/mmwr.mm6609e1

2. Maricar N, Callaghan MJ, Felson DT, O'Neill TW. Predictors of response to intra-articular steroid injections in knee osteoarthritis--a systematic review. Rheumatology (Oxford). 2013; 52(6):1022-1032. https://doi.org/10.1093/rheumatology/kes368

3. Raeissadat SA, Rayegani SM, Forogh B, Hassan Abadi P, Moridnia M, Rahimi Dehgolan S. Intra-articular ozone or hyaluronic acid injection: Which one is superior in patients with knee osteoarthritis? A 6-month randomized clinical trial. Journal of pain research. 2018; 11:111-117. https://doi.org/10.2147/JPR.S142755 
4. Raeissadat SA, Rayegani SM, Ahangar AG, Abadi PH, Mojgani P, Ahangar OG. Efficacy of Intra-articular Injection of a Newly Developed Plasma Rich in Growth Factor (PRGF) Versus Hyaluronic Acid on Pain and Function of Patients with Knee Osteoarthritis: A Single-Blinded Randomized Clinical Trial. Clin Med Insights Arthritis Musculoskelet Disord. 2017; 10:1179544117733452. https:// doi.org/10.1177/1179544117733452

5. Manoto SL, Maepa MJ, Motaung SK. Medical ozone therapy as a potential treatment modality for regeneration of damaged articular cartilage in osteoarthritis. Saudi journal of biological sciences. 2018; 25(4):672-679.https://doi.org/10.1016/j.sjbs.2016.02.002

6. Karouzakis E, Neidhart M, Gay RE, Gay S. Molecular and cellular basis of rheumatoid joint destruction. Immunology letters. 2006; 106(1):8-13. https://doi.org/10.1016/j.imlet.2006.04.011

7. Vaillant JD, Fraga A, Diaz MT, Mallok A, Viebahn Hansler R, Fahmy Z et al. Ozone oxidative postconditioning ameliorates joint damage and decreases pro-inflammatory cytokine levels and oxidative stress in PG/PS-induced arthritis in rats. European journal of pharmacology. 2013; 714(1-3):318-324. https://doi.org/10.1016/j.ejphar.2013.07.034

8. Lopes de Jesus CC, Dos Santos FC, de Jesus L, Monteiro I, Sant'Ana M, Trevisani VFM. Comparison between intra-articular ozone and placebo in the treatment of knee osteoarthritis: A randomized, double-blinded, placebo-controlled study. PloS one. 2017; 12 (7):e0179185. https://doi.org/10.1371/journal.pone.0179185

9. Arias-Vazquez PI, Tovilla-Zarate CA, Hernandez-Diaz Y, Gonzalez-Castro TB, Juarez-Rojop IE, Lopez-Narvaez ML et al. Short-Term Therapeutic Effects of Ozone in the Management of Pain in Knee Osteoarthritis: A Meta-Analysis. PM \& R: the journal of injury, function, and rehabilitation. 2019; 11(8):879-887. https://doi.org/10.1002/pmrj.12088

10. Noori-Zadeh A, Bakhtiyari S, Khooz R, Haghani K, Darabi S. Intra-articular ozone therapy efficiently attenuates pain in knee osteoarthritic subjects: A systematic review and meta-analysis. Complementary therapies in medicine. 2019; 42:240-247. https://oi. org/10.1016/j.ctim.2018.11.023

11. Dernek B, Kesiktas FN. Efficacy of combined ozone and platelet-rich-plasma treatment versus platelet-rich-plasma treatment alone in early stage knee osteoarthritis. Journal of back and musculoskeletal rehabilitation. 2019; 32(2):305-311. https://doi.org/10.3233/ BMR-181301

12. Duymus TM, Mutlu S, Dernek B, Komur B, Aydogmus S, Kesiktas FN. Choice of intra-articular injection in treatment of knee osteoarthritis: platelet-rich plasma, hyaluronic acid or ozone options. Knee Surg Sports Traumatol Arthrosc. 2017; 25(2):485-492. https://doi.org/10.1007/s00167-016-4110-5

13. Kohn MD, Sassoon AA, Fernando ND. Classifications in Brief: Kellgren-Lawrence Classification of Osteoarthritis. Clin Orthop Relat Res. 2016; 474(8):1886-1893.https://doi.org/10.1007/s11999-016-4732-4

14. Madrid Declaration On Ozone Therapy. https://www.drsozone.com/wp-content/uploads/2014/02/Madrid-Declaration-updated-July-30. pdf. Accessed.

15. Basaran S, Guzel R, Seydaoglu G, Guler-Uysal F. Validity, reliability, and comparison of the WOMAC osteoarthritis index and Lequesne algofunctional index in Turkish patients with hip or knee osteoarthritis. Clin Rheumatol. 2010; 29(7):749-756. https://doi.org/10.1007/ s10067-010-1398-2

16. Xing D, Xu Y, Liu Q, Ke Y, Wang B, Li Z et al. Osteoarthritis and all-cause mortality in worldwide populations: grading the evidence from a meta-analysis. Scientific reports. 2016; 6:24393. https://doi.org/10.1038/srep24393

17. Chang KV, Hung CY, Aliwarga F, Wang TG, Han DS, Chen WS. Comparative effectiveness of platelet-rich plasma injections for treating knee joint cartilage degenerative pathology: a systematic review and meta-analysis. Archives of physical medicine and rehabilitation. 2014; 95(3):562-575. https://doi.org/10.1016/j.apmr.2013.11.006 